\title{
Sociodemographic Inequalities and Health Behaviors in the Dental and Periodontal Health
}

\author{
${ }^{1}$ Rachid Ait Addi, ${ }^{2}$ Abdelhafid Benksim, ${ }^{3}$ Mohamed Cherkaoui
}

\begin{abstract}
Aim: Dental decay and periodontal diseases are very common allover the world with outcomes ranging from dental loss to systemic diseases. In this study, we aim to determine the impact of sociodemographic disparities and health behaviors on dental and periodontal health (DPH).
\end{abstract}

Materials and methods: An epidemiological study was conducted amongst 600 dental patients in two private dental clinics in the region of Marrakesh, Morocco from 2012 to 2015. Three hundred seventeen patients between 25 years and 46 years old who were contacted by telephone to carry out a supplementary survey and of these 317 patients, 82 subjects were maintained. A questionnaire was followed to assess sociodemographic disparities, self-reported oral health status, and hygiene behaviors.

Results: The age of the sample varies between 3 years and 78 years with an average of $31.2 \pm 14.3$ years. Also, the dominant age group is between 26 and 44 years old with a percentage of $47.6 \%$. The distribution of the population by sex shows good equity ( $53.4 \%$ of men and $46.6 \%$ of women). For the educational level, $7.3 \%$ of the population is illiterate, $17.1 \%$ have a primary level, $39.0 \%$ have a high school level and $36.6 \%$ have a university level. In addition, habitation is urban at $79.3 \%$, and rural at $20.7 \%$. Furthermore, we note that only $28.8 \%$ of patients have a good daily toothbrushing frequency which is equal to or greater than 3 .

Conclusion: Dental and periodontal health (DPH) are indeed affected by sociodemographic and economic status with higher dental problems among rural and poor people.

Clinical significance: Dental and periodontal diseases are serious problems of public health allover the world.

The lack of oral education and dental cares, particularly in developing countries and rural areas may increase these affections. The result of this study shows the implication of sociodemographic disparities and health behaviors in dental and periodontal outcomes.

This work will complement the limited knowledge of the existing interaction between sociodemographic disparities and health behaviors and dental and periodontal affections. It is also a fundamental starting of public health preventive or therapeutic strategies.

\footnotetext{
${ }^{1}$ Private Dental Clinic Al AMAL, Kelaa des Sraghna, Morocco

${ }^{2}$ Department of Biology, Laboratory of Human Ecology, School of Sciences Semlalia, Cadi Ayyad University, Marrakech. Morocco

${ }^{3} \mathrm{High}$ Institute of Nursing and Technical Health, Marrakech, Morocco

Corresponding Author: Rachid Ait Addi, Department of Biology, Laboratory of Human Ecology, School of Sciences Semlalia, Cadi Ayyad University, Marrakech. Morocco, Phone: 212 661350175, e-mail: dr.rachid.aitaddi@gmail.com
}

Keywords: Epidemiology, Periodontitis, Sociodemography, Tooth decay.

How to cite this article: Addi RA, Benksim A, Cherkaoui M. Sociodemographic Inequalities and Health Behaviors in the Dental and Periodontal Health. World J Dent 2019;10(1):29-34.

Source of support: Nil

Conflict of interest: None

\section{INTRODUCTION}

Affecting about $40-50 \%$ of adults in industrialized countries, tooth decay is an infectious disease due to the interaction of biological, behavioral and socioeconomic influences. ${ }^{1,2}$

The development of caries is multifactorial, depending on many interacting variables to promote its development especially bacteria, alimentation, the host's oral environment, and time. ${ }^{3}$

Periodontitis is an inflammatory disease affecting the supporting tissues of the tooth. In the general US population, studies found that minority and lower-income groups have poorer oral health. ${ }^{4-6}$

Moreover, healthy habits and good oral hygiene, including twice-daily toothbrushing, are important to prevent gum disease and maintain good oral health. ${ }^{7}$

In addition, the prevalence of less than twice tooth brushing per day is higher among people in low and middle income than in high income and also the prevalence of less than an annual dental check-up is higher among people $\mathrm{s}$ in developing countries.

Few studies have been conducted on oral health based on the files of dental clinics patients, in particular in low and middle-income countries. Therefore, this epidemiologic study describes the DPH of a sample of dental patients, examines relationships between sociodemographic factors and (DPH), and behavioral factors and (DPH). The study involved the exploitation of 600 client files randomly selected from two private dental offices in the Marrakech region in Morocco from 2012 to 2015.

\section{MATERIALS AND METHODS}

\section{Ethics Statement}

The participants were questioned after having the full explanation of the purpose of our study and receiving their verbal consent. The consent procedure was approved 
by the health ministry delegation. Also, our study has been independently reviewed and approved by the health ministry delegation. We note that we have not yet a local ethics committee because of that the health ministry delegation ensures that function. Finally, our research has been conducted in full accordance with the World Medical Association Declaration of Helsinki.

\section{Consulting Methods}

The study involved the exploitation of 600 client files randomly selected from two private dental offices in the region of Marrakesh in Morocco from 2012 to 2015.

These files contain information like name, age, sex, health status, address, phone, profession, and dental and periodontal interventions. Thereafter, we selected among them 317 files of patients between 25 years and 46 years old, who were contacted by telephone to carry out a supplementary survey. Of these 317 patients, 82 subjects were maintained. Their distribution by sex was as follows: 41 women and 41 men.

These 82 patients were interviewed using a questionnaire that includes sociodemographic information (age, sex, profession, habitation, educational level, health insurance, and socioeconomic status), height, weight, health behaviors( daily brushing frequency, type of toothbrush, brushing method, and flossing), and self-reported dental and periodontal status.

To assess the oral status of patients, we used frequencies of presence/absence of gingivitis, periodontitis, gingival bleeding, halitosis, gingival recession, tooth mobility, tooth decay, oral abscess, and tooth loss. Also, patients were categorized according to their dental status: degraded state when the number of treated teeth is greater than or equal to three and normal when the number of affected teeth is less than 3 .

Age was coded into four classes $(<18,18-25,26-45$, and over 45 ), also the level of education has been coded into four classes (illiterate, primary, middle or high school). Professions have been classified into two categories: favored and disfavored.

Additionally, the following variables were used to assess dental hygiene:

- Daily brushing frequency with two modalities $(\geq 3$ times/day and <3 times/day).

- Type of a toothbrush with two modalities (soft and medium or hard)

- Brushing method with two modalities (right method and another method).

- Flossing modalities (yes and no).

Finally, the body mass index (BMI) was calculated which allowed us to classify subjects into two classes (underweight or normal and overweight or obese).

\section{Exclusion Criteria}

Smoking patients and patients with general diseases were excluded from the study.

\section{Statistical analysis}

Data was entered and analyzed by statistical package for social sciences (SPSS) ver. 13.0 (Chicago, IL, USA). Independent sample t-tests were used and were considered statistically significant at $p$ values below 0.05 .

\section{RESULTS}

Table 1 gives a description of the population studied by sociodemographic and economic characteristics.

The age of the sample varies between 3 years and 78 years with an average of $31.2 \pm 14.3$ years. Also, the dominant age group is between 26 years and 44 years old with a percentage of $47.6 \%$. The distribution of the population by sex shows good equity $(53.4 \%$ of men and $46.6 \%$ of women). For the educational level, $7.3 \%$ of the population is illiterate, $17.1 \%$ have a primary level, $39.0 \%$ have a high school level, and 36.6\% have a university level.

The habitation is urban at $79.3 \%$, and rural at $20.7 \%$.

Regarding the social system, about $46.3 \%$ have no insurance, $3.7 \%$ have RAMED $^{*}, 14.6 \%$ have $\mathrm{CNOPS}^{* *}$, and $35.6 \%$ have other types of coverage.

Regarding the socioeconomic level, $71.4 \%$ of the sample was favored and $28.6 \%$ disfavored.

The body mass index calculated on the population aged between 25 and 46 shows that $1.4 \%$ of this sample

Table 1: Sociodemographic and economic characteristics of the population

\begin{tabular}{llll}
\hline Variables & Modalities & Effective & Percentage (\%) \\
\hline Age & $<18$ & 68 & 13.8 \\
& $18-25$ & 111 & 22.6 \\
& $26-44$ & 241 & 49.6 \\
& $\geq 45$ & 72 & 14.6 \\
\hline Sex & Male & 304 & 53.4 \\
& Female & 265 & 46.6 \\
\hline Habitation & Urban & 65 & 79.3 \\
& Rural & 17 & 20.7 \\
\hline Level of education & Illiterate & 6 & 7.3 \\
& Primary & 14 & 17.1 \\
& High school & 32 & 39.0 \\
& University & 30 & 36.6 \\
\hline Health insurance & RAMED & 3 & 3.7 \\
& CNOPS & 12 & 14.6 \\
& Others & 29 & 35.4 \\
& Without & 38 & 46.3 \\
\hline Socioeconomic status & Optimal & 14 & 28.6 \\
& Poor & 35 & 71.4 \\
\hline
\end{tabular}

RAMED: A public insurance dedicated to people with poor incomes CNOPS: A public insurance dedicated to the employees of the state Others: private insurances 
are severely underweight, $51.4 \%$ present underweight moderate, $34.8 \%$ are at a normal weight, and $12.4 \%$ are at overweight or obese.

Table 2 contains some data relating to dental hygiene.

Only $28.8 \%$ of patients have a good daily toothbrushing frequency which is equal to or greater than 3.

The study also found that $59.2 \%$ of the population uses the correct method of brushing. Furthermore, 57.0\% of the population uses medium or hard toothbrushes.

Finally, only $14.1 \%$ use dental flossing.

Table 3 gives the relationship between dental hygiene factors and oral problems.

The results show that patients who brush with medium or hard brushes have more dental problems (dental mobility) than those who brush with soft toothbrushes. Also, patients who brush their teeth with the right method have less gingivitis than patients using other methods.

Graphs 1 shows the relationship between socioeconomic factors and oral problems.

No significant differences were observed depending on the sex.

The education level shows differences in dental caries with a benefit in favor of higher levels. For the professions, the differences are important mainly for gingival bleeding, which is more pronounced in the disfavored population.

\section{DISCUSSION}

The oral health study aimed to describe (DPH) and highlight the different risk factors. Based on the results, the 26-44 age group is the most group who visited the dentist.

$93.9 \%$ of this age category estimated to have oral problems which often dental losses, caries, and gum bleeding. In another study involving adolescents from the Marrakesh area aged 12-18, Kaoutar et al. (2013) found a prevalence rate of caries involvement estimated at $44.1 \%{ }^{8}$

In addition, the study shows no significant difference in DPH according to sex which is contradictory to the study conducted by Eke et al. who found that men develop more periodontal diseases than women $(56.4 \%$ vs. $38.4 \%$ ). This high risk of developing periodontal diseases is explained by another survey which found that women are more using professional dental care and have higher oral hygiene than men., ${ }^{910}$

Inversely, women develop more dental caries than men $(92.66 \%$ vs. $90.57 \%)$. This high caries prevalence among women is due to the earlier eruption of teeth in girls, the longer exposure to the cariogenic oral environment, better access to food supplies and snacks during pregnancy. ${ }^{11}$

Moreover, the results show that subjects with a level of education that does not exceed high school have a high percentage of caries compared to subjects with a higher level of education. This may be due to the awareness of educated people to the importance of oral health. It is in common with the study conducted by Vano et al. who found a positive association between the educational level and dental and periodontal status. ${ }^{12}$

Depending on the socioeconomic level, the disfavored class has a poorer oral health status than the favored class. This result seems logical as subjects with a high socioeconomic level have the means to take care of their general health and in particular oral health. Thus, it is similar to the study of Piovesan et al. who found that socioeconomic inequalities at individual and community levels are major determinants for the occurrence of the oral diseases, and Chung et al. who found that people with disadvantaged circumstances had a significant proportion of dental disease. ${ }^{13,14}$

In addition, the results show a higher average of dental caries in rural people than urban people. Rural living is related to poverty, illiteracy, poor dental status and no health insurance coverage. These factors have an impact on oral health care, service delivery, and research. ${ }^{15-17}$

Furthermore, the study shows no significative differences in oral health between different (BMI) classes, contrarily to previous studies which found that increased BMI has been associated with increased gingival bleeding. ${ }^{18}$

Table 2: Characteristics of health behaviors in the population

\begin{tabular}{|c|c|c|c|c|}
\hline Variables & $\mu \pm \sigma$ & Modalities & Effective & $\%$ \\
\hline \multirow{2}{*}{ Daily brushing frequency } & \multirow{2}{*}{$1,96 \pm 0,82$} & $>=3$ times $/$ day & 21 & 28.8 \\
\hline & & $<3$ times/day & 52 & 71.2 \\
\hline \multirow{3}{*}{ Type of toothbrush } & & Soft & 30 & 42.3 \\
\hline & & Medium & 36 & 50.7 \\
\hline & & Hard & 5 & 7.0 \\
\hline \multirow{2}{*}{ Brushing method } & & The right method & 42 & 59.2 \\
\hline & & Othermethod & 29 & 40.8 \\
\hline \multirow{2}{*}{ Flossing } & & Yes & 11 & 14.1 \\
\hline & & No & 67 & 85.9 \\
\hline
\end{tabular}




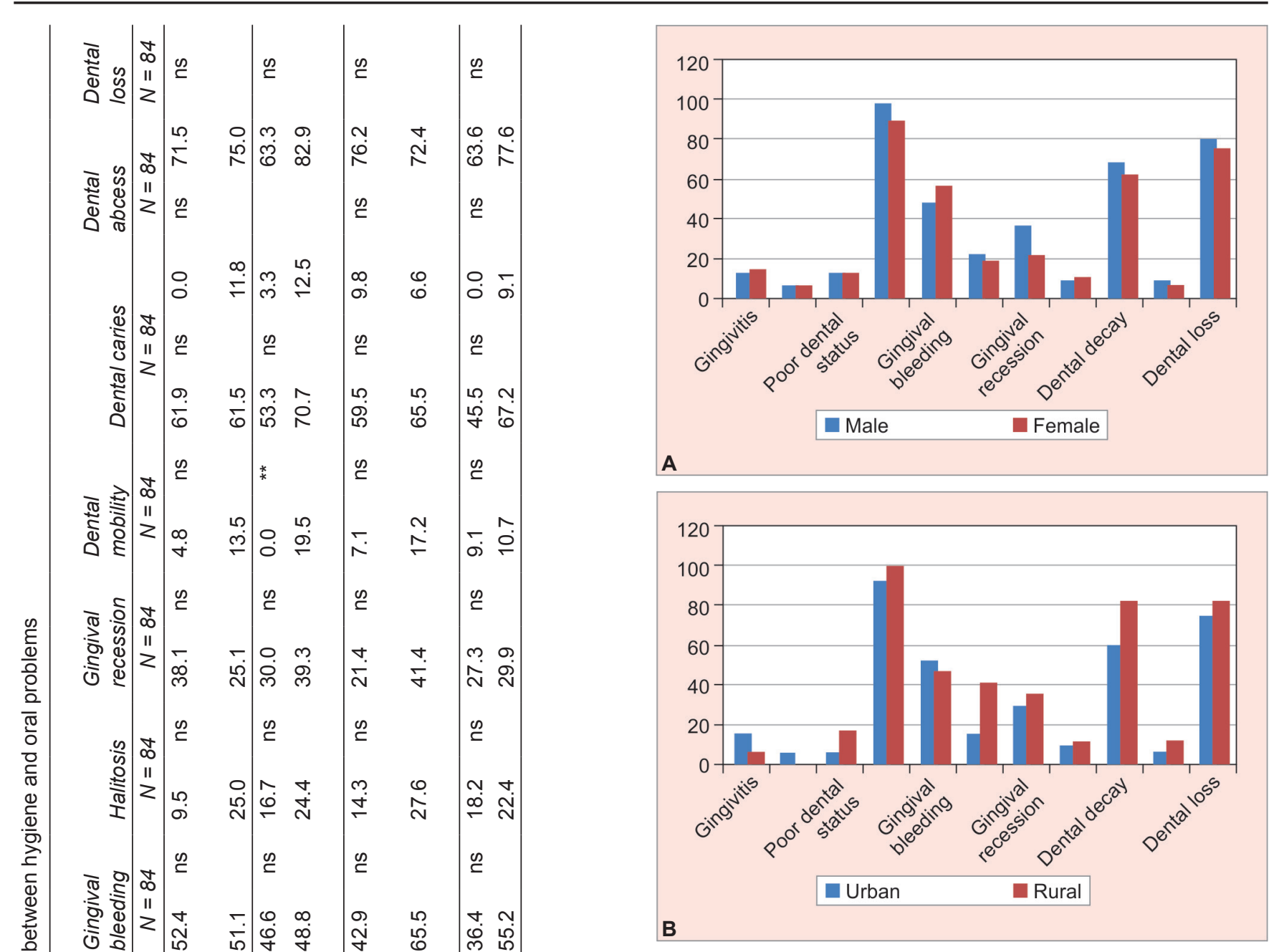

Graphs $1 A$ and $B:$ The relationship between socioeconomic factors and oral problems: (A) Sex and oral problems; (B) Habitation and oral problems

This may be because (BMI) has a positive relationship with proinflammatory markers and an inverse relationship with antioxidants. ${ }^{19,20}$ Moreover, the associations between low socioeconomic status and being overweight have been demonstrated. ${ }^{21}$

\section{CONCLUSION}

The study showed that dental problems are quite frequent among the population of the region of Marrakeh.

Dental and periodontal health (DPH) is intimately linked at the sociodemographic and economic level; dental problems are higher among rural people, lower education levels and lower economic classes. Also, health behaviors such a correct toothbrush may prevent gingivitis. Indeed, it is essential to conduct preventive and therapeutic programs as well as promoting dental health awareness, especially among poor, rural, and less-equated people.

\section{ACKNOWLEDGMENT}

Authors want to thank Pr Loubna Bahije, Professor at the School of Dentistry of RABAT, Morocco for her scientific support. 

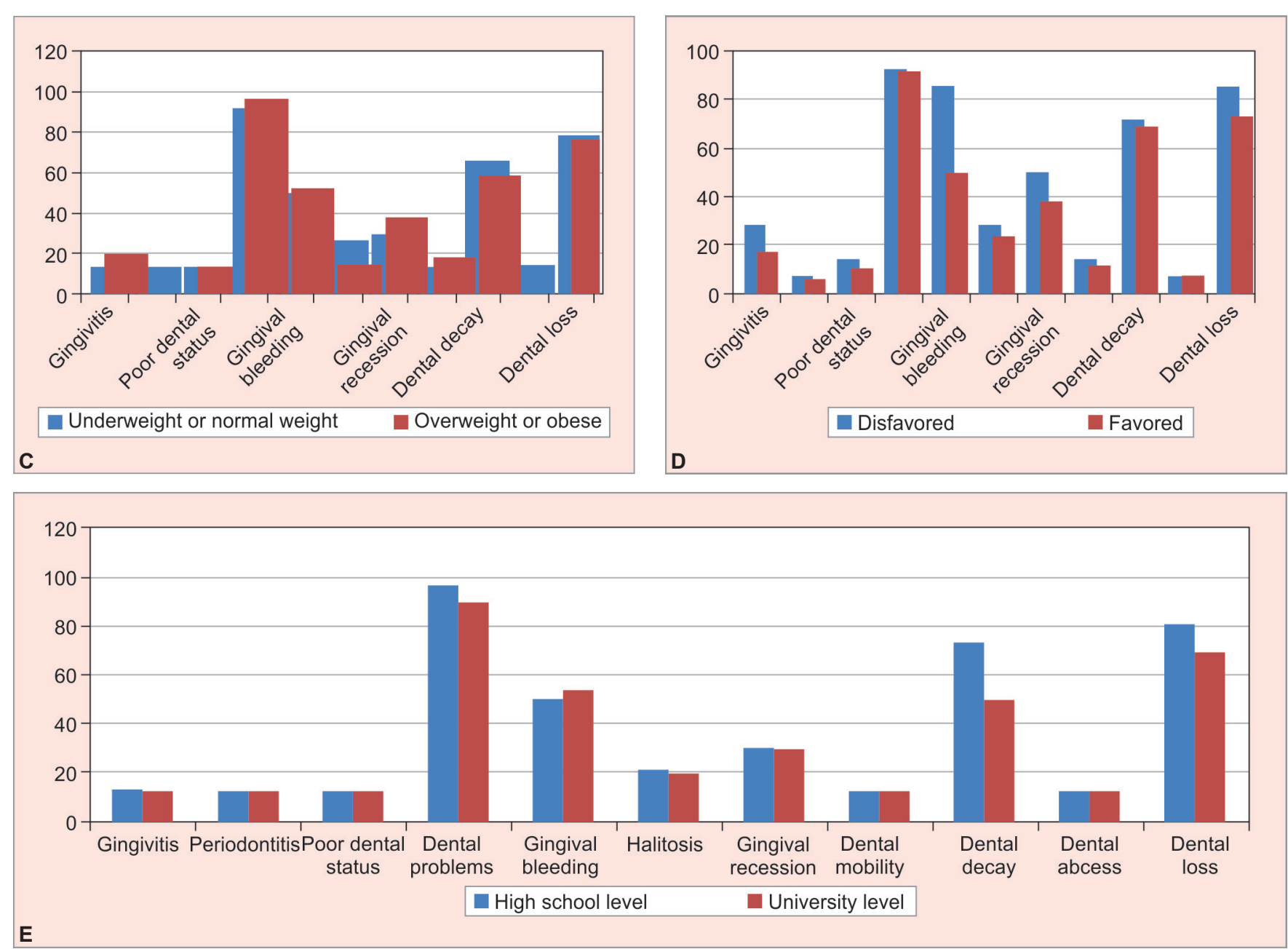

Graphs 1C to E: The relationship between socioeconomic factors and oral problems: (C) Profession and oral problems; (D) IMC and oral problems (E) Level of education and oral problems

\section{REFERENCES}

1. Brown LJ, Wall TP, Lazar V. Trends in caries among adults 18 to 45 years old. J Am Dent Assoc 2002;133:827-834.

2. Lukacs JR, Largaespada L L. Explaining sex differences in dental caries prevalence: saliva, hormones, and life history etiologies. American Journal of Human Biology 2006;18(4):540-555.

3. Fejerskov O, Nyvad B, Kidd E, eds. Dental caries: the disease and its clinical management. 3rd ed. Hoboken, NJ: Wiley Blackwell; 2015.

4. Dye BA, Tan S, Smith V, Lewis BG, Barker LK, Thornton-Evans G, et al. Trends in oral health status: United States, 1988-1994 and 1999-2004. National Center for Health Statistics Vital Health Stat. 2007;11(248):1-92.

5. Eke PI, Dye BA, Wei L, Thornton-Evans GO, Genco RJ. Prevalence of periodontitis in adults in theUnited States: 2009 and 2010. J Dent Res. 2012;91(10):914-920.

6. Grembowski D, Spiekerman C, Milgrom P. Social gradients in dental health among low-income mothers and their young children. J Health Care Poor Underserved. 2012;23(2): 570-588.

7. Peltzer K, Pengpid S. Oral Health Behaviour and Social and Health Factors in University Students from 26 Low, Middle and High Income Countries. Int. J. Environ. Res. Public Health (2014);12247-12260.
8. Kaoutar K, Hilali MK, Loukid M.La situation de la carie dentaire chez les adolescents de la Wilaya de Marrakech (Maroc). Antropo 2013;29:101-108.

9. Eke PI, Dye BA, Wei L, Slade GD, Thornton-Evans GO, Borgnakke WS, Taylor GW, Page RC, Beck JD, Genco RJ. Update on Prevalence of Periodontitis in Adults in the United States: NHANES 2009 to 2012. J Periodontol. 2015;86:611-622.

10. Buunk-Werkhoven YAB, Buunk AP. Fear of social rejection and oral self-care in men versus women. Int Dent J. 2015;65 (Suppl1):1-57.

11. Mamai-Homata E, Koletsi-Kounari H, and MargaritisV. Gender differences in oral health status and behavior of Greek dental students: A meta-analysis of 1981, 2000, and 2010 data. J Int Soc Prev Community Dent. 2016 Jan-Feb;6(1):60-68.

12. Vano M, Gennai S, Karapetsa D, et al. The influence of educational level and oral hygiene behaviours on DMFT index and CPITN index in an adult Italian population: an epidemiological study. Int J Dent Hyg. 2015 May;13(2):151-157.

13. Piovesan C, Mendes FM, Antunes JLF, Ardenghi, TM. Inequalitie sin the distribution of dental caries among 12-year-old Brazilian school children. Braz Oral Res (2011);25: 6975.

14. Chung LH, Gregorich SE, Armitage GC, Gonzalez-Vargas J, Adams SH. Sociodemographic disparities and behavioral factors in clinical oral health status during pregnancy. Community Dent Oral Epidemiol. 2014;42:151-159. 
15. Ahn S, Burdine JN, Smith ML, Ory MG, Phillips CD. Residential rurality and oral health disparities: influences of contextual and individual factors. J Prim Prev.( 2011) Feb;32(1): 29-41.

16. OgunbodedeEO, Kida IA, Madjapa HS, Amedari M, Ehizele A, et al. Oral Health Inequalities between Rural and Urban Populations of the African and Middle East Region. Adv Dent Res. 2015 Jul;27(1):18-25.

17. Peltzer K, Pengpid S. Oral Health Behaviour and Social and Health Factors in University Students from 26 Low, Middle and High Income Countries. Int. J. Environ. Res. Public Health 2014;11:12247-12260.
18. Ritchie CS, Kinane DF. Nutrition, inflammation, and periodontal disease. Nutrition 2003;19:475-476.

19. Hermsdorff HH, Puchau B, Volp AC, Barbosa KB, Bressan J, Zulet MÁ, et al. Dietary total antioxidant capacity is inversely related to central adiposity as well as to metabolic and oxidative stress markers in healthy young adults. Nutr Metab (Lond) 2011;8:59.

20. Franchini R, Petri A, Migliaro M, Rimondini L. Poor oral hygiene and gingivitis are associated with obesity and overweight status in paediatric subjects. J Clin Periodontol 2011;38: 1021-1028.

21. Östberg AL, Bengtsson C, Lissner L, Hakeberg M. Oral health and obesity indicators. BMC Oral Health 2012;12:50. 